\title{
CT Densitometry as a Predictor of Pulmonary Function in Lung Cancer Patients
}

\author{
Fiachra Moloney ${ }^{*}$, , Sebastian McWilliams ${ }^{1}$, Lee Crush ${ }^{1}$, Patrick D. Mc Laughlin ${ }^{1}$, \\ Marcus P. Kennedy ${ }^{2}$, Michael Henry ${ }^{2}$, O.J. O’ Connor ${ }^{1}$ and Michael M. Maher ${ }^{1}$ \\ ${ }^{I}$ Department of Radiology, Cork University Hospital and University College Cork, Cork, Ireland \\ ${ }^{2}$ Department of Respiratory Medicine, Cork University Hospital, Cork, Ireland
}

Open Access

\begin{abstract}
Purpose: Preoperative pulmonary assessment is undertaken in patients with resectable lung cancer to identify those at increased risk of perioperative complications. Guidelines from the American College of Chest Physicians indicate that if the $\mathrm{FEV}_{1}$ and DLCO are $\geq 60 \%$ of predicted, patients are suitable for resection without further evaluation.

The aim of our study is to determine if quantitative measures of lung volume and density obtained from pre-operative CT scans correlate with pulmonary function tests. This may allow us to predict pulmonary function in patients with lung cancer and identify patients who would tolerate surgical resection.
\end{abstract}

Materials and Methods: Patients were identified retrospectively from the lung cancer database of a tertiary hospital. Image segmentation software was utilized to estimate total lung volume, normal lung volume (values $-500 \mathrm{HU}$ to $-910 \mathrm{HU}$ ), emphysematous volume (values less than $-910 \mathrm{HU}$ ), and mean lung density from pre-operative CT studies for each patient and these values were compared to contemporaneous pulmonary function tests.

Results: A total of 77 patients were enrolled. FEV1 was found to correlate significantly with the mean lung density $(\mathrm{r}=.762, \mathrm{p}<.001)$ and the volume of emphysema $(\mathrm{r}=-.678, \mathrm{p}<.001)$. DLCO correlated significantly with the mean lung density $(\mathrm{r}=.648, \mathrm{p}<.001)$ and the volume of emphysematous lung $(\mathrm{r}=-.535, \mathrm{p}<.001)$.

Conclusion: The results of this study suggest that both $\mathrm{FEV}_{1}$ and DLCO correlate significantly with volume of emphysema and mean lung density. We now plan to prospectively compare these CT parameters with measures of good and poor outcome postoperatively to identify CT measures that may predict surgical outcome preoperatively.

Keywords: Computed tomography, lung density, pulmonary function.

\section{INTRODUCTION}

Lung cancer is one of the leading causes of death from cancer worldwide. There was an estimated 222,520 new cases of lung cancer in the United States in 2010, with 157,300 deaths [1]. The 5-year survival rate varies with TNM stage, from $77 \%$ (stage pIA) to $2 \%$ (stage cIV) for patients with local and advanced stage disease respectively [2].

Surgical resection and radical radiotherapy remain the only curative options for patients with localized non-small cell lung cancer (NSCLC). However, only $20-25 \%$ of patients have surgically resectable disease at the time of diagnosis. Mortality rates of $5.4 \%$ and $3.2 \%$ are reported for pneumonectomy and lobectomy respectively $[3,4]$.

Preoperative pulmonary assessment is performed in patients with resectable disease to identify those at increased risk of perioperative complications and to establish baseline pulmonary function prior to exposure to potential pneumotoxic chemo- and radiotherapy.

*Address correspondence to this author at the Department of Radiology, Cork University Hospital, Wilton, Cork, Ireland; Tel: 00353861938625 ; Fax:+00 35321 4922857; E-mail: fiachramoloney@hotmail.com
Guidelines from the American College of Chest Physicians indicate that the forced expiratory volume in one second $\left(\mathrm{FEV}_{1}\right)$ and the diffusing capacity of carbon monoxide (DLCO) should be measured initially. If the $\mathrm{FEV}_{1}$ and DLCO are $\geq 60 \%$ of predicted, patients are at low risk of complications and can undergo resection without further testing. However if the $\mathrm{FEV}_{1}$ or DLCO are $<60 \%$ predicted, further evaluation with a quantitative lung scan or cardiopulmonary exercise testing is recommended [5].

The aim of our study is to determine if quantitative measures of lung volume and density obtained from preoperative CT scans correlate with pulmonary function tests. This may allow us to predict pulmonary function in patients with lung cancer and identify patients who would tolerate surgical resection.

\section{MATERIALS AND METHODS}

\section{Patients}

Patients were identified retrospectively from the lung cancer database of a tertiary hospital. Inclusion criteria were the availability of a CT thorax and pulmonary function tests (PFT's) from May 2009 to April 2010. Exclusion criteria were previous pneumonectomy, lobectomy, and pleural effusion. 
Patients with radiological evidence of pulmonary fibrosis on CT were also excluded.

Ethical approval was granted by the Clinical Research Ethics Committee of our institution.

\section{Computed Tomography}

CT thorax scans were acquired using a 64-slice GE Medical Systems Lightspeed Volume CT (GE Healthcare, Wisconsin, USA) or a 4-slice Toshiba Aquilion multidetector row CT (Toshiba America Medical Systems, Tustin, CA, USA). After infusion of intravenous contrast media, the chest region was scanned during breath-hold at the end of inspiration in the supine position. Automated bolus tracking software was used to trigger image acquisiton with a threshold of $100 \mathrm{HU}$ in a region of interest placed over the thoraic aorta.

Lung density and volume measurements were performed using the OsiriX digital analysis programme (OsiriX Imaging Software, v3.7.1, OsiriX Foundation, Geneva). Image segmentation software was used to segment lung parenchyma according to predetermined density thresholds. Lung attenuation threshold limits of -500 to -1024 HU were used to exclude soft tissue surrounding the lungs. Areas with attenuation values less than -910 HU were considered representative of poorly functioning emphysematous lung.

Following this, quantitative measures of volume and density of each segment were calculated. The following CT parameters were calculated for each patient: total lung volume (TLV), the volume of normal lung parenchyma (values $-500 \mathrm{HU}$ to $-910 \mathrm{HU}$ ), the volume of emphysematous lung (values less than $-910 \mathrm{HU}$ ), and the mean lung density (see Fig. 1). The regions highlighted in green represent lung that has an attenuation value of less than $-910 \mathrm{HU}$ and is considered to represent emphysematous lung.

\section{Pulmonary Function Testing}

All pulmonary function tests were performed in a dedicated pulmonary function laboratory. Spirometric values including forced expiratory volume in 1 second $\left(\mathrm{FEV}_{1}\right)$, forced vital capacity ( $\mathrm{FVC}$ ), $\mathrm{FEV}_{1} / \mathrm{FVC}$ ratio, the mean midexpiratory flow between $75 \%$ and $25 \%$ of the FVC (MMEF 75-25), maximum expiratory flow at $50 \%$ of forced vital capacity $\left[\mathrm{MEF}{ }_{50}\right]$, peak expiratory flow at 50\% (PEF), and forced expiratory time (FET) were measured using a Jaeger Masterscreen Pneumo spirometer (Jaegar/HP/Dell; San Diago, CA, USA).

The diffusion capacity of the lung for carbon monoxide [DLCO], and the DLCO corrected for alveolar volume were measured with a Jaegar Masterscreen diffusion analyzer (Jaegar/HP/Dell; San Diago, CA, USA). All pulmonary function data are presented as a percentage of predicted values for the patient's age, gender and height [11].

\section{Statistical Analysis}

Statistical analyses of the data were performed using the Statistical Package for the Social Sciences software (SPSS version 19.0; SPSS; Chicago, IL, USA).

Univariate correlations were examined using Pearson's product-moment correlation coefficient or Spearman's rank correlation in the case of nonnormally distributed data.

All tests were two-tailed and a p-value $<0.05$ was considered statistically significant. Data are expressed as means $\pm \mathrm{SD}$.

\section{RESULTS}

92 patients were identified for inclusion in the study. 15 patients were excluded due to pleural effusion $(n=8), C T$ evidence of pulmonary fibrosis $(n=4)$, previous pneumonectomy $(n=2)$, or lobectomy $(n=1)$. In total, 77 patients were included in the study with a mean age of $68 \pm$ 12 years (range, 30-88 years). 41 patients had a histological diagnosis of adenocarcimona, 20 of squamous cell carcinoma, and 16 of small cell carcimona. 44 patients in the cohort were current smokers with 15 ex-smokers. 54 patients $(70 \%)$ were male. The mean $\mathrm{FEV}_{1}$ (\% of predicted) was $88.1 \pm 24.3$ and the mean DLCO (\% of predicted) was $56.5 \pm$ 21.5.
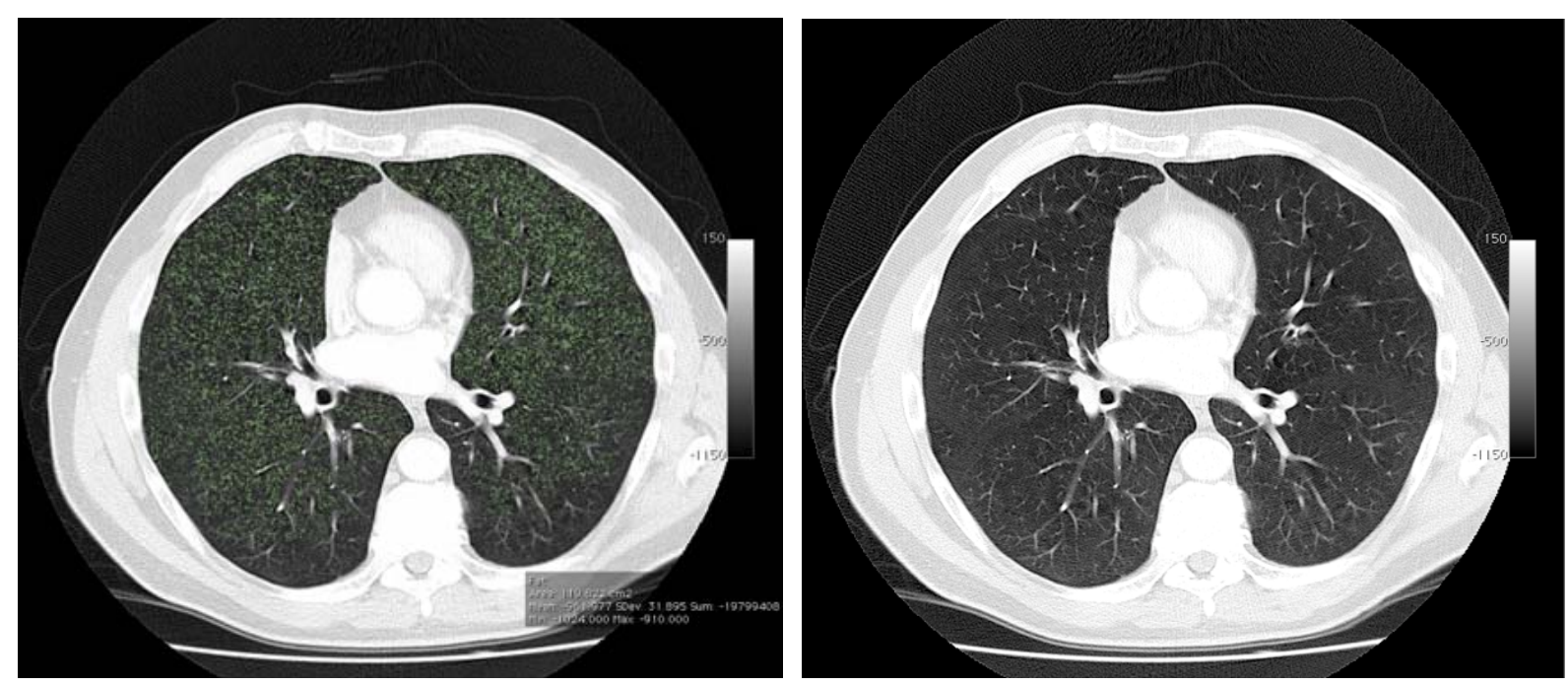

Fig. (1). Chest CT (axial slice) of a 64 year-old male patient.All lung parenchyma with an attenuation value of less than -910 HU is highlighted in green (left image) using image segmentation software. The volume of the region of interest (green areas) is then calculated. The corresponding slice is shown on the right prior to application of our technique. 


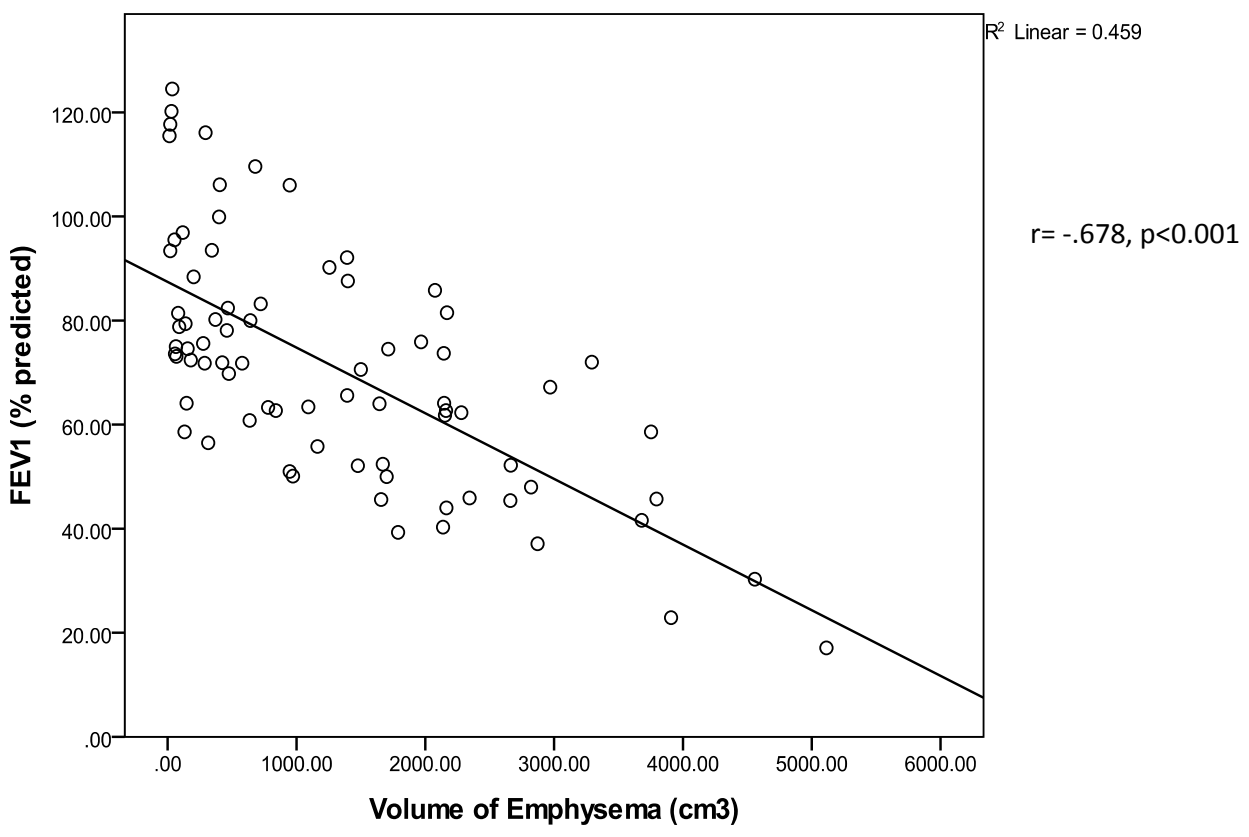

Fig. (2). Correlation between forced expiratory volume in the first second $\left(\mathrm{FEV}_{1}-\%\right.$ of predicted $)$ and the volume of emphysema $\left(\mathrm{cm}^{3}\right)(\mathrm{r}=-$ $.678, \mathrm{p}<0.001)$

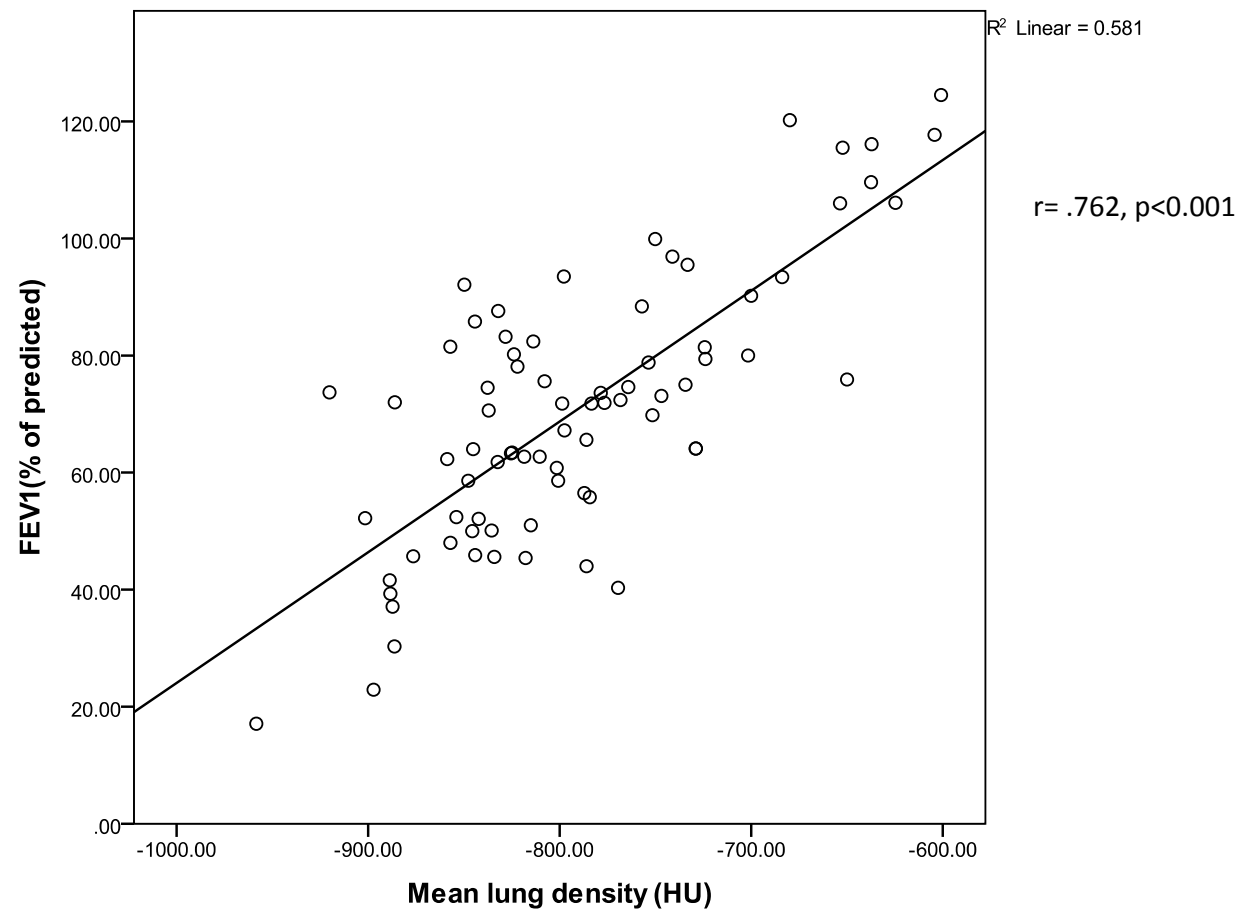

Fig. (3). Correlation between the forced expiratory volume in the first second ( $\mathrm{FEV}_{1}-\%$ of predicted) and the mean lung density (HU). ( $\mathrm{r}=$ $.762 ; \mathrm{p}<0.001)$.

$\mathrm{FEV}_{1}$ (\% of predicted) correlated significantly with the volume of emphysema (values less than $-910 \mathrm{HU})(\mathrm{r}=-.678$, $\mathrm{p}<0.001$ ) (see Fig. 2) and the mean lung density ( $\mathrm{r}=.762$, p $<0.001$ ) (see Fig. 3). DLCO (\% of predicted) correlated with the mean lung density $(r=.648, p<0.001)$ (see Fig. 4) and the volume of emphysema $(\mathrm{r}=-.535, \mathrm{p}<0.001)$ (see Fig. 5).

Table 1 shows the strengh of association of the CT measures with other pulmonary function indices (see Table 1).

\section{DISCUSSION}

Lung cancer is the leading cause of cancer death in the United States. Surgical resection is the treatment of choice for patients with localised non-small cell carcinoma. The incidence of complications varies, depending on the extent of the resection, the pulmonary reserve of the patient, and the presence of comorbid factors. 


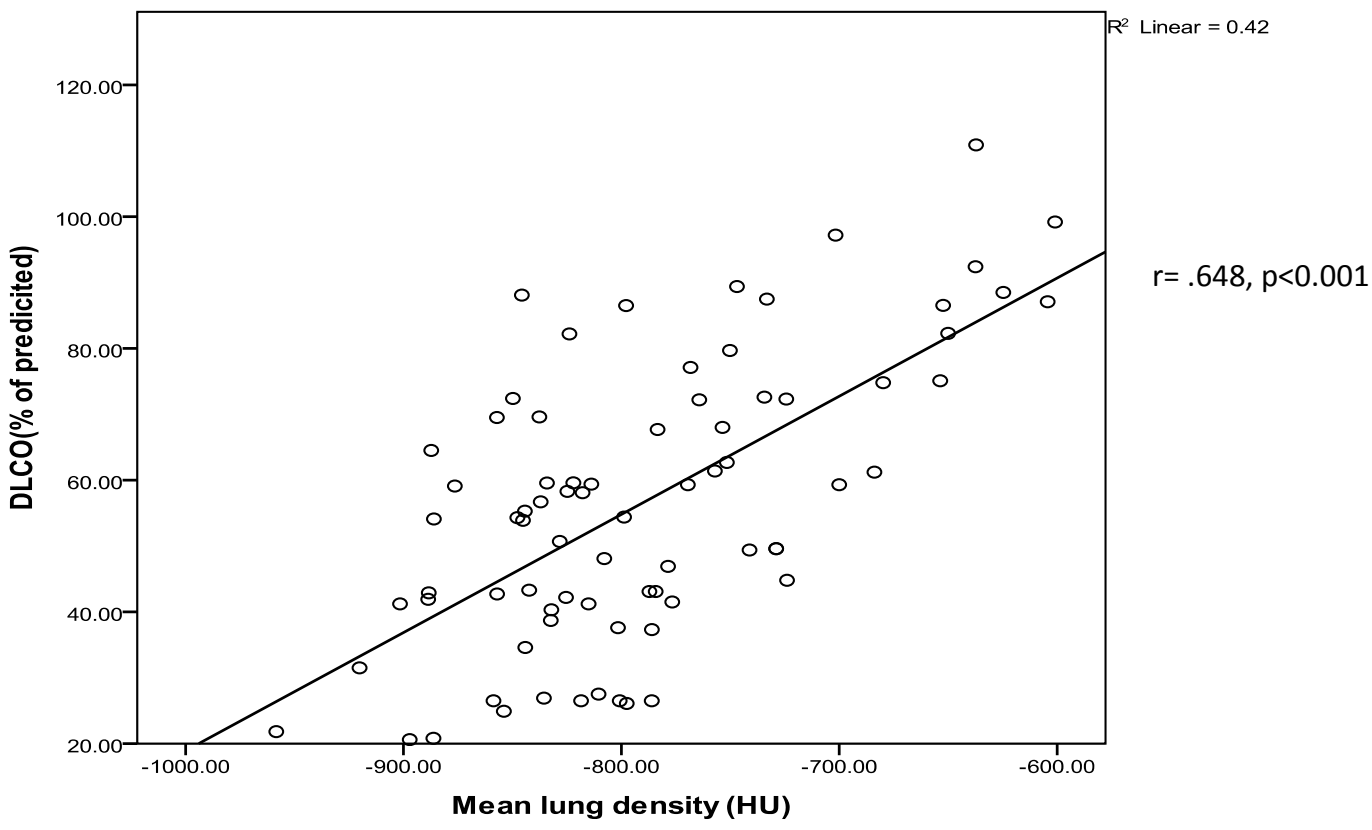

Fig. (4). Correlation between diffusion capacity for carbon monoxide (DLCO - $\%$ of predicted) and the mean lung density ( $r=.648$; $p<0.001$ ).

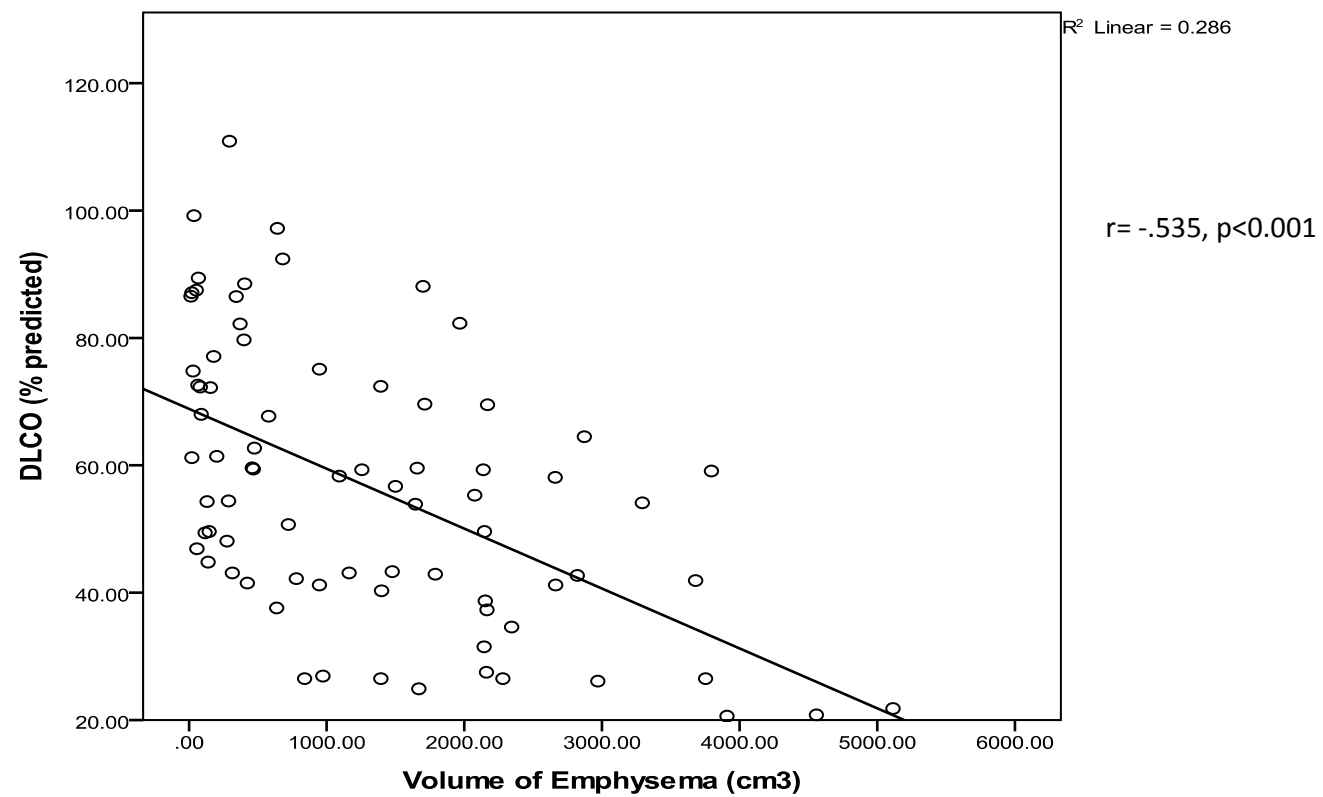

Fig. (5). Correlation between diffusion capacity for carbon monoxide (DLCO - $\%$ of predicted) and the volume of emphysema $\left(\mathrm{cm}^{3}\right)(\mathrm{r}=-$ $.535 ; \mathrm{p}<0.001)$.

Preoperative risk-stratification of potential candidates is essential prior to surgery. Pulmonary assessment is especially important as patients with lung cancer are more likely to be smokers and have airways disease.

Emphysema is characterised by destruction of the airways distal to the terminal bronchioles as evident by areas of abnormally low attenuation on CT. A lung attenuation threshold of $-910 \mathrm{HU}$ was chosen to differentiate low attenuation areas of emphysema from normal lung parenchyma after a review of the literature. Several attenuation thresholds have been quoted by different studies for the recogniton of emphysematous lung ranging from -910 to $-950 \mathrm{HU}$ [6-10]. Muller et al found good correlation with macroscopic emphysema using an attenuation threshold of $910 \mathrm{HU}$ in patients who had received intravenous contrast [9]. All of the patients in our study received intravenous contrast prior to image acquisition. Although contrast medium increases the attenuation of lung parenchyma, we choose an attenuation threshold that had been found by previous studies to correlate well with emphysema on CT in patients who had received intravenous contrast as part of their scan protocal. Moreover, other studies that omit intravenous contrast from the scan protocal report similar attenuation thresholds in the range of -900 to -910 to segment emphsematous lung [7]. 
Table 1. Correlation Between Pulmonary Function Measures and CT Parameters

\begin{tabular}{|c|c|c|c|c|}
\hline & TLV & Mean Density & Volume (-900 to $-500 \mathrm{HU})$ & Volume $(<-900 \mathrm{HU})$ \\
\hline FVC $\%$ of predicted & $\mathrm{r}=-.051, \mathrm{p}=.661$ & $\mathrm{r}=-.047, \mathrm{p}=.687$ & $\mathrm{r}=.003, \mathrm{p}=.983$ & $\mathrm{r}=.050, \mathrm{p}=.665$ \\
\hline MMEF ${ }_{75-25 \%} \%$ of predicted & $\mathrm{r}=-.187, \mathrm{p}=.104$ & $\mathrm{r}=.284, \mathrm{p}=.012$ & $\mathrm{r}=.191, \mathrm{p}=.096$ & $\mathrm{r}=-.270, \mathrm{p}=.018$ \\
\hline MEF $_{50 \%} \%$ of predicted & $\mathrm{r}=-.211, \mathrm{p}=.066$ & $\mathrm{r}=.342, \mathrm{p}=.002$ & $r=.231, p=.043$ & $\mathrm{r}=-.329, \mathrm{p}=.003$ \\
\hline FET & $\mathrm{r}=.343, \mathrm{p}=.002$ & $r=-.311, p=.005$ & $\mathrm{r}=-.058, \mathrm{p}=.614$ & $\mathrm{r}=.334, p=.003$ \\
\hline DLCO/VA $\%$ of predicted & $\mathrm{r}=-.245, \mathrm{p}=.032$ & $\mathrm{r}=.376, \mathrm{p}=.001$ & $\mathrm{r}=.150, \mathrm{p}=.194$ & $\mathrm{r}=-.246, \mathrm{p}=.031$ \\
\hline
\end{tabular}

Given is the correlation coefficient $\mathrm{r}$, as well as the $\mathrm{p}$ value. Statistically significant data $(\mathrm{p}<0.05)$ are highlighted in bold type. $\mathrm{FVC}$ : forced vital capacity; $\mathrm{FEV}$ : forced expiratory volume in one second; MMEF ${ }_{75-25 \%}$ : mean mid-expiratory flow between $75 \%$ and $25 \%$ of the FVC; MEF $50 \%$ : maximum expiratory flow at $50 \%$ of the FVC; PEF: peak expiratory flow; FET: forced expiratory time; DLCO/VA: diffusion capacity for carbon monoxide corrected for alveolar volume.

We choose to omit patients with a history of pneumonectomy, lobectomy, and pleural effusion on CT as this would lead to erronous measures of lung volume. Furthermore, patients with CT signs of pulmonary fibrosis were excluded due to the volume reducing effect of restrictive lung disease.

Our findings are in keeping with previous studies that report significant correlation between areas of lung with low attenuation and spirometric measures of pulmonary function. As observed by several studies, both $\mathrm{FEV}_{1}$ and DLCO were found to correlate significantly with the volume of emphysema [8, 9, 11-15].

Moreover, DLCO was found to correlate with mean lung density in this study. Failure of $\mathrm{FEV}_{1}$ and DLCO to correlate with total lung volume may be due to the fact that we lacked normal reference values of lung volume in size-matched controls. However, exposing healthy individuals to radiation for this purpose is unjustified.

We now plan to prospectively compare these CT parameters with measures of good and poor outcome postoperatively in patients undergoing lung surgery. This may allow us to accurately determine CT measurements that could be used as a predictor of surgical outcome preoperatively and identify patients who would tolerate surgical resection. We are not proposing that CT replaces pulmonary function testing but both modalities are potentially complimentary. In time, with improvements in CT densitometry, CT may obviate the need for pulmonary function testing in selected patients, resulting in reduced healthcare costs and delays prior to surgery. It must be stressed that these measures of lung volume and density are obtained from clinically indicated pre-operative staging CT scans in patients with lung cancer and do not require any modification of imaging protocol, additional exposure to ionising radiation or additional scanning time.

We recognise the limitations of our study. Our cohort consisted largely of patients with mild to moderate emphysema. Thus our results may not be applicable to patients with severe disease. We plan to further study the use of these techniques in this clinical setting, in a prospective trial with larger numbers of lung cancer patients to determine if our results retain their validity. Although image aquisiition was performed during breath-hold at the end of inspiration, it is possible that some of the patients did not or were unable to comply which would alter measures of lung volume and density. Although patients with features of fibrosis on CT were excluded, patients with early subtle changes or spirometric evidence of fibrotic lung disease may have inadvertently been included. Furthermore, it is likely that our correlations would not hold true for patients who also have co-exisitng fibrotic lung disease.

It is also worth noting that the strength of assocation observed between the CT measures and DLCO/VA were not as strong as those obseved with DLCO. The explanation for this is a unclear.

In conclusion, the results of this study suggest that both $\mathrm{FEV}_{1}$ and DLCO correlate significantly with volume of emphysema and mean lung density. We now plan to prospectively compare these $\mathrm{CT}$ parameters with measures of good and poor outcome postoperatively to identify CT measures that may predict surgical outcome preoperatively.

\section{CONFLICT OF INTEREST}

The authors confirm that this article content has no conflicts of interest.

\section{ACKNOWLEDGEMENTS}

Declared none.

\section{REFERENCES}

[1] American Cancer Society. Cancer Facts and Figures 2010. Atlanta: American Cancer Society 2010.

[2] Rami-Porta R, Ball D, Crowley J, et al. The IASCL lung cancer staging project: proposals for the revision of the $\mathrm{T}$ descriptors in the forthcoming (seventh) edition of the TNM classification of lung cancer. J Thorac Oncol 2007; 2: 593-602.

[3] Powell E, Pearce A, Cook D, et al. UK pneumonectomy outcome study (UKPOS): a prospective observational study of pneumonectomy outcome. J Cardiovasc Surg 2009; 4: 41.

[4] Licker M, de Perrot M, Hohn L, et al. Perioperative mortality and major cardiopulmonary complications after lung surgery for nonsmall cell carcinoma. Eur J Cardiothoracic Surg 1999; 15: 314-9.

[5] Datta D, Lahiri B. Preoperative evaluation of patients undergoing lung resection surgery. Chest 2003; 123: 2096-103.

[6] Pauls S, Gulkin D, Feuerlein S, et al. Assessment of COPD severity by computed tomography: correlation with lung functional testing. Clin Imaging 2010; 34: 172-8.

[7] Arakawa A, Yamashita Y, Nakayama Y, et al. Assessment of lung volumes in pulmonary emphysema using multidetector helical CT: comparison with pulmonary function tests. Comput Med Imaging Graph 2001; 25: 399-404. 
[8] Sashidhar K, Gulati M, Gupta D, et al. Emphysema in heavy smokers with normal chest radiography. Detection and quantification by HRCT. Acta Radiologica 2002; 43: 60-5.

[9] Muller L, Staples C, Miller R, et al. Density mask. An objective method to quantitate emphysema using computed tomography. Chest 1988; 94: 782-87.

[10] Litmanovich D, Boiselle P, Bankier A. CT of pulmonary emphysema - current status, challenges, and future directions. Eur Radiol 2009; 19: 537-51.

[11] Pellegrino R, Viegi G, Brusasco V, et al. American Thoracic Society/European Respiratory Society task force: standardisation of lung function testing. Eur Resp J 2005; 26: 948-68.
[12] Bernspang E, Diaz S, Stoel B, et al. CT lung densitometry in young adults with alpha-1-antitrypsin deficiency. Open Respir Med J 2011; 105(1): 74-9.

[13] Desai SR, Hansell DM, Walker A, et al. Quantification of emphysema: a composite physiologic index derived from CT estimation of disease extent. Eur Radiol 2007; 17: 911-8.

[14] Cerveri I, Dore R, Corisco A, et al. Assessment of emphysema in COPD: a functional and radiological study. Chest 2004; 125: 17148.

[15] Sanders C, Nath PH, Bailey WC, et al. Detection of emphysema with computed tomography: correlation with pulmonary function tests and chest radiography. Invest Radiol 1988; 23: 262-6.

(C) Moloney et al.; Licensee Bentham Open.

This is an open access article licensed under the terms of the Creative Commons Attribution Non-Commercial License (http://creativecommons.org/licenses/by-nc/3.0/) which permits unrestricted, non-commercial use, distribution and reproduction in any medium, provided the work is properly cited. 